\title{
The Relationship Between Lipid Profile and Outcomes of Pregnant Women with Gestational Diabetes Mellitus and Hypertensive Disorders: A Retrospective Study
}

\author{
Yuan Li \\ 0003-0438-7794 \\ Wenqing Chen \\ Nanjing University Medical School Affiliated Nanjing Drum Tower Hospital \\ Xiaoqian Wang \\ Nanjing University Medical School Affiliated Nanjing Drum Tower Hospital \\ Fengjuan Jiang \\ Nanjing University Medical School Affiliated Nanjing Drum Tower Hospital \\ Xiaotian Chen ( $\nabla$ xttchen@163.com ) \\ https://orcid.org/0000-0001-6112-0784
}

Nanjing University Medical School Affiliated Nanjing Drum Tower Hospital https://orcid.org/0000-

Research article

Keywords: Lipids, pregnancy, gestational diabetes mellitus, hypertensive disorders, clinical outcome

Posted Date: June 2nd, 2020

DOI: https://doi.org/10.21203/rs.3.rs-29209/v1

License: (c) (i) This work is licensed under a Creative Commons Attribution 4.0 International License.

Read Full License 


\section{Abstract}

Background Dyslipidemia during pregnancy in women with gestational diabetes mellitus (GDM) and hypertensive disorders of pregnancy (HDP) has been extensively studied in western countries. However, similar researches have rarely been conducted in Asia. The present study aimed to evaluate maternal lipid profile changes in relation to pregnancy complications and clinical outcomes.

Methods The medical records of 668 pregnant women were retrospectively analyzed. Maternal fasting serum lipids were assayed for total cholesterol (TC), triglycerides (TG), high-density lipoproteincholesterol (HDL-C), low-density lipoprotein-cholesterol (LDL-C), apolipoprotein A1 (Apo A1) and Apo B concentrations during the second trimesters. The atherogenic index of plasma (AIP) was calculated. The pregnancy complications included GDM and HDP. The clinical outcomes were collected by evaluating delivery mode, postpartum hemorrhage, macrosomia, birth weight, body length and neonatal Apgar score.

Results Levels of TG and AIP were elevated while decreased TC and HDL-C was observed in women with GDM compared with that of the control group $(p<0.05)$. TG and AIP in HDP group were higher than in control group $(p<0.05)$. Significant differences were observed in postpartum hemorrhage, birth weight and body length between control group and women with GDM or HDP $(p<0.05)$. Compared with women with serum $T G \geq 2.5 \mathrm{mmol} / \mathrm{L}$ or $T C \geq 6.2 \mathrm{mmol} / \mathrm{L}$, the incidence of GDM, cesarean section and macrosomia was lower in normal lipid group $(p<0.05)$. Spearman correlation analysis showed that the incidence of GDM was positively correlated with levels of TG $(r=0.226, p<0.01)$ and AIP $(r=0.250, p<$ $0.01)$, while negative correlated with TC $(r=-0.106, p<0.01)$ and HDL-C $(r=-0.190, p<0.01)$. AIP was correlated positively with the incidence of $\operatorname{HDP}(r=0.115, p=0.043)$. According to the logistic regression analysis, each unit elevation in AIP increased the risk of GDM by 6.943 times $(\mathrm{OR}=6.943,95 \% \mathrm{Cl}$ : $1.761-$ 27.374).

Conclusions Lipid control, especially for women with GDM and HDP, might be a potential strategy for improving the pregnancy outcomes.

\section{Background}

Maternal energy metabolism in the second half of pregnancy is directed toward lipolysis, which indicates a physiological adaptation of mothers to maintain stable fuel supplementation to the fetus. Mildly increased lipidemia occurs in early pregnancy, with a more pronounced elevation by the second and third trimesters. However, it is still difficult to ascertain which level of lipid elevation is physiological or pathological nowadays. There is no consensus on which criteria should be used in diagnosis of hyperlipidemia during gestation in China.

Pregnancy suffers a substantial increase in the workload of different organs, which may more likely to result in metabolic diseases. Gestational diabetes mellitus (GDM) is a common metabolic complication in pregnant women and affects up to $22 \%$ of pregnancies [1]. Moreover, patients with gestational and preGDM have a greater risk for hypertensive disorders of pregnancy (HDP), which is a major cause of 
maternal mortality worldwide [2]. Although clinical and experimental studies have suggested that GDM and HDP share pathophysiological features, such as endothelial injury and dyslipidemia $[3,4]$. Limited studies have assessed the concurrent risk factors for GDM and HDP.

Hyperlipidaemia is a well recognized risk factor for metabolic syndromes. It has been reported that an abnormal elevation of lipids during pregnancy exerts adverse impacts on pregnant women and newborns. Previous studies indicate that levels of triglycerides (TG) are significantly increased in women with GDM and HDP compared to those without these metabolic diseases $[5,6]$. Nevertheless, the role of lipids profiles in predicting GDM and HDP is a controversial issue [7, 8]. Hyperlipidaemia is relation to an adverse intrauterine environment and fetal overgrowth. Recent studies show that disturbance in maternal levels of TG and HDL-C was an independent risk factor for macrosomia[9]. However, the relationship between maternal lipid level and birth weight is still inconsistent.

Currently, the use of lipid-lowering drugs during pregnancy is relatively contraindicated. Thus evaluating the diagnostic criteria of hyperlipidemia and its association with adverse pregnancy outcomes was urgently needed, which may allow for appropriate and timely intervention. In the present study, we examined the blood lipids in patients with GDM and HDP, and analyzed putative relations with pregnancy complications and clinical outcomes.

\section{Methods}

\section{Study population}

Pregnant women who were admitted to Nanjing Drum Tower Hospital and delivered between January 2015 and December 2017 were recruited for the present study. The inclusion criteria were as follows: 1) age between 20 to 40 years, 2) live-born singleton pregnancy, 3) naturally conceived, 4) gestation at 2028 gestational weeks, 5) physician-diagnosed GDM and HDP, 6) and had integrated medical records. We excluded those with other pregnancy complications, medical or surgical diseases (i.e a specific psychiatric disorder, immunological, liver and kidney diseases), abortive times $\geq 3$, smoking, alcohol abuse and long-term medication treatment (i.e anti-hyperglycemic, anti-hyperlipidemic and immunosuppressive drugs). Finally, 668 pregnant women ( $n=273$ in control group, $n=359$ in GDM group, $n=36$ in HDP group) were enrolled in the final analysis.

\section{Data collection}

Data were extracted from medical records. General characteristics of the study population included maternal age, pre-pregnancy body mass index (BMI), gravidity, parity, residence, monthly income and education attainment. Maternal metabolic parameters included serum TG, TC, HDL-C, LDL-C Apo A1 and Apo B. AIP $=\log (T G / H D L)$, and the abnormal value was set at AIP $>0.15$. Other clinical data were collected by evaluating delivery mode, postpartum hemorrhage, macrosomia, birth weight, body length and neonatal Apgar score. 


\section{Diagnosis of hyperlipidemia}

There is no consensus on which criteria should be used in diagnosis of hyperlipidemia. We combined the diagnostic criteria of dyslipidemia in general Chinese population (2016 revised edition) and clinical data, the diagnostic criteria for hyperlipidemia of second and third trimester was established: $\triangle T C>$ $6.20 \mathrm{mmol} / \mathrm{L}$ or $\otimes T G>2.30 \mathrm{mmol} / \mathrm{L}$.

\section{Statistical analysis}

To ensure the normal distribution of variables, Histogram and Kolmogrov-Smirnov test were applied. Differences between groups were compared using the independent two sample t test, a rank sum test and chi-squared test. Pearson correlation coefficient was used to evaluate correlations. Logistic regression analysis was performed to identify risk factors that were significantly correlated with GDM and HDP. TG, TC and AIP, which were statistically significant according to univariate logistic regression, were regarded as confounding variables in the multivariable logistic regression model. All statistical analyses were performed using SPSS statistical software (Chicago, IL, USA). A p value $<0.05$ was considered as statistically significant.

\section{Results}

A total of 668 pregnant women were included in analyses. Of all of women, 359 were GDM, 36 were HDP and the remaining 273 were regarded as controls. Table 1 shows the general characteristics of the study group. Compared with the control group, maternal demographic and clinical characteristics in GDM and HDP groups, including age, pre-pregnancy BMI, gravidity, parity, residence, monthly income and education attainment, were not statistically different (Table 1). 
Table 1

Characteristics of the study population (means $\pm S D, n \%$ )

\begin{tabular}{|c|c|c|c|c|}
\hline & $\begin{array}{l}\text { Control group } \\
(n=273)\end{array}$ & $\begin{array}{l}\text { GDM group } \\
(n=359)\end{array}$ & $\begin{array}{l}\text { HDP group } \\
(n=36)\end{array}$ & $P$ \\
\hline Age (years) & $30.04 \pm 4.02$ & $30.66 \pm 4.22$ & $30.86 \pm 3.83$ & 0.140 \\
\hline Gestational weeks & $21.86 \pm 2.36$ & $21.73 \pm 2.30$ & $21.91 \pm 3.04$ & 0.740 \\
\hline pre-pregnancy BMI & $22.85 \pm 3.12$ & $23.52 \pm 3.60$ & $23.77 \pm 4.10$ & 0.671 \\
\hline Gravidity (n, \%) & & & & 0.767 \\
\hline 1 & $120(44.0)$ & 148 (41.2) & $16(44.4)$ & \\
\hline$\geq 2$ & $153(56)$ & $211(58.8)$ & $20(55.6)$ & \\
\hline Parity (n, \%) & & & & 0.525 \\
\hline$\leq 1$ & $178(65.2)$ & $229(63.8)$ & $20(55.6)$ & \\
\hline$\geq 2$ & $95(34.8)$ & $130(36.2)$ & $16(44.4)$ & \\
\hline Residence (n, \%) & & & & 0.147 \\
\hline City & 240 (87.9) & $332(92.5)$ & $33(91.7)$ & \\
\hline Rural area & $33(12.1)$ & $27(7.5)$ & $3(8.3)$ & \\
\hline Monthly income (n, \%) & & & & 0.879 \\
\hline$\leq 6000$ & $88(32.2)$ & $120(33.4)$ & $13(36.1)$ & \\
\hline$\varangle 6000$ & $185(67.8)$ & $239(66.6)$ & $23(63.9)$ & \\
\hline Education attainment $(\mathrm{n}, \%)$ & & & & 0.826 \\
\hline$\leq$ Secondary & $130(47.6)$ & $176(49.0)$ & $19(52.8)$ & \\
\hline$\geq$ College & $143(52.4)$ & $183(51.0)$ & $17(47.2)$ & \\
\hline Smoking (n, \%) & - & - & & - \\
\hline Alcohol intake (n, \%) & - & - & & - \\
\hline
\end{tabular}

Maternal lipid profile in the second trimester can be found in Table 2. Overall, women with GDM had higher levels of TG $(2.38 \pm 1.37$ versus $2.05 \pm 0.97 \mathrm{mmol} / \mathrm{L}, \mathrm{P}=0.001)$, AIP $(0.08 \pm 0.24$ versus $-0.02 \pm$ $0.20, P<0.001)$ and lower levels of TC $(5.39 \pm 0.94$ versus $5.59 \pm 0.96 \mathrm{mmol} / \mathrm{L}, \mathrm{P}=0.010)$ and HDL-C $(1.86 \pm 0.42$ versus $2.02 \pm 0.41 \mathrm{mmol} / \mathrm{L}, \mathrm{P}<0.001)$ than that of control. Similar differences in TG $(2.53 \pm$ 1.29 versus $2.05 \pm 0.97 \mathrm{mmol} / \mathrm{L}, P=0.036)$ and $\operatorname{AIP}(0.08 \pm 0.27$ versus $-0.02 \pm 0.20, P=0.043)$ were observed between HDP and control groups. LDL-C, Apo A1 and Apo B were not significantly different between the groups. Regarding the pregnancy outcomes, there was more excessive postpartum 
hemorrhage in both GDM and HDP groups than that in control group (446.17 \pm 264.82 versus $397.27 \pm$ $213.61 \mathrm{ml}, \mathrm{P}=0.012 ; 478.14 \pm 242.99$ versus $397.27 \pm 213.61 \mathrm{ml}, \mathrm{P}=0.036)$. As for the conditions of newborn, the birth weight was even lighter in the GDM and HDP groups (3362.63 \pm 443.63 versus $3445.95 \pm 519.96 \mathrm{~g}, \mathrm{P}=0.030 ; 3161.39 \pm 774.94$ versus $3445.95 \pm 519.96 \mathrm{~g}, \mathrm{P}=0.039$ ). Meanwhile, body length of the newborn in these two groups was slightly shorter compared with the control group $(50.08 \pm$ 1.91 versus $50.43 \pm 1.75 \mathrm{~cm}, \mathrm{P}=0.016 ; 48.75 \pm 3.19$ versus $50.43 \pm 1.75 \mathrm{~cm}, \mathrm{P}=0.038$ ). In addition, we failed to find significant differences in mean changes of Apgar score (1 $\mathrm{min}$ ) between the groups.

Table 2

The levels of lipid indicators and pregnancy outcomes (Means \pm SD)

\begin{tabular}{|llllll|}
\hline & $\begin{array}{l}\text { Control group } \\
(\mathbf{n}=\mathbf{2 7 3})\end{array}$ & $\begin{array}{l}\text { GDM group } \\
(\mathbf{n}=\mathbf{3 5 9})\end{array}$ & $\mathbf{P} *$ & $\begin{array}{l}\text { HDP group } \\
(\mathbf{n}=\mathbf{3 6})\end{array}$ & $\mathbf{P}$ \# \\
\hline TG (mmol/L) & $2.05 \pm 0.97$ & $2.38 \pm 1.37$ & 0.001 & $2.53 \pm 1.29$ & 0.036 \\
\hline $\mathrm{TC}(\mathrm{mmol} / \mathrm{L})$ & $5.59 \pm 0.96$ & $5.39 \pm 0.94$ & 0.010 & $5.46 \pm 1.21$ & 0.456 \\
\hline HDL-C (mmol/L) & $2.02 \pm 0.41$ & $1.86 \pm 0.42$ & 0.000 & $1.91 \pm 0.43$ & 0.122 \\
\hline LDL-C (mmol/L) & $2.69 \pm 0.75$ & $2.61 \pm 0.73$ & 0.159 & $2.55 \pm 0.86$ & 0.284 \\
\hline Apo A (g/L) & $2.42 \pm 0.41$ & $2.37 \pm 0.41$ & 0.195 & $2.43 \pm 0.42$ & 0.806 \\
\hline Apo B (g/L) & $1.06 \pm 0.31$ & $1.04 \pm 0.22$ & 0.486 & $1.05 \pm 0.35$ & 0.833 \\
\hline AlP & $-0.02 \pm 0.20$ & $0.08 \pm 0.24$ & 0.000 & $0.08 \pm 0.27$ & 0.043 \\
\hline $\begin{array}{l}\text { Postpartum hemorrhage } \\
\text { (ml) }\end{array}$ & $397.27 \pm$ & $446.71 \pm$ & 0.012 & $478.14 \pm$ & 0.036 \\
\hline Birth weight (g) & 213.61 & 264.82 & & 242.99 & \\
\hline Body length (cm) & $3445.95 \pm$ & $3362.63 \pm$ & 0.030 & $3161.39 \pm$ & 0.039 \\
\hline Apgar 1 min & 519.96 & 443.63 & & 774.94 & \\
\hline
\end{tabular}


Table 3

The effect of the serum lipid on clinical outcomes (n, \%)

\begin{tabular}{|llll|}
\hline & $\begin{array}{l}\text { Normal lipid } \\
(\mathbf{n = 3 4 7 )}\end{array}$ & $\begin{array}{l}\text { Hyperlipidemia } \\
(\mathbf{n = 3 2 1 )}\end{array}$ & P-value \\
\hline GDM & $164(47.3)$ & $195(60.7)$ & 0.000 \\
\hline HDP & $17(4.9)$ & $19(5.9)$ & 0.560 \\
\hline Cesarean section & $97(28.0)$ & $113(35.2)$ & 0.044 \\
\hline Postpartum hemorrhage & $101(29.1)$ & $81(25.2)$ & 0.261 \\
\hline Macrosomia & $25(7.2)$ & $38(11.8)$ & 0.041 \\
\hline
\end{tabular}

Table 4

Association between GDM and lipid levels

\begin{tabular}{|lllll|}
\hline Factors & \multicolumn{2}{l}{ GDM group } & \multicolumn{2}{l|}{ HDP group } \\
\cline { 2 - 5 } & $\mathbf{r}$ & $\mathbf{P}$ & $\mathbf{r}$ & $\mathbf{P}$ \\
\hline TG & 0.226 & 0.000 & 0.101 & 0.077 \\
\hline TC & -0.106 & 0.008 & -0.055 & 0.334 \\
\hline HDL & -0.190 & 0.000 & -0.095 & 0.097 \\
\hline LDL & -0.049 & 0.216 & -0.075 & 0.189 \\
\hline Apo A & -0.054 & 0.176 & 0.010 & 0.866 \\
\hline Apo B & 0.013 & 0.748 & -0.035 & 0.535 \\
\hline AIP & 0.250 & 0.000 & 0.115 & 0.043 \\
\hline
\end{tabular}

Maternal hyperlipidemia was defined as $\triangle \mathrm{TC}>6.20 \mathrm{mmol} / \mathrm{L}$ or $\triangle \mathrm{TG}>2.30 \mathrm{mmol} / \mathrm{L}$. When grouped by lipid measurement, the incidence of GDM ( 47.3 versus $60.7 \%, P<0.01)$, cesarean section $(28.0$ versus $35.2 \%$, $P=0.044)$ and macrosomia (7.2 versus $11.8 \%, P=0.041)$ was significantly increased in the hyperlipidemia group. No statistically significant difference was seen between the two groups in terms of the rate of HDP and postpartum hemorrhage $(P>0.050)$.

We investigated whether there was an association between maternal serum lipids and the risk of GDM or HDP. Using Spearman correlation analysis, we found that the incidence of GDM was positively correlated with levels of TG $(r=0.226, p<0.01)$ and AIP $(r=0.250, p<0.01)$, while negative correlated with TC $(r=$ $-0.106, p<0.01)$ and HDL $(r=-0.190, p<0.01)$. The change of AIP was correlated positively with the incidence of $\operatorname{HDP}(r=0.115, p=0.043)$. We then performed a logistic regression in order to see the effect of serum lipids on GDM and HDP. We identified several confounders that might be correlated with the presence of GDM and found that maternal TG, TC and AIP were statistically significant. Therefore, these 
variables were regarded as confounders in the multivariable logistic model. Table 5 shows that the risk of GDM increased approximately 6.943-fold with each unit elevation in AIP (OR $=6.943,95 \%$ Cl: 1.761 27.374). However, results from the logistic regression indicated that lipids and other variables were almost no contribution to risk of HDP.

Table 5

Logistic regression analysis of GDM and lipid levels

\begin{tabular}{|c|c|c|c|c|}
\hline \multirow[t]{2}{*}{ Factors } & \multicolumn{4}{|l|}{ GDM } \\
\hline & B & $\mathbf{P}$ & OR & $95 \% \mathrm{Cl}$ \\
\hline TG & 0.010 & 0.947 & 1.010 & $0.751 \sim 1.358$ \\
\hline TC & -0.182 & 0.050 & 0.833 & $0.696 \sim 0.997$ \\
\hline HDL & 0.008 & 0.570 & 1.008 & $0.980 \sim 1.038$ \\
\hline LDL & -0.153 & 0.160 & 0.858 & $0.693 \sim 1.062$ \\
\hline Apo A & -0.255 & 0.195 & 0.775 & $0.528 \sim 1.139$ \\
\hline Apo B & -0.207 & 0.487 & 0.813 & $0.453 \sim 1.459$ \\
\hline AIP & 1.938 & 0.006 & 6.943 & $1.761 \sim 27.374$ \\
\hline
\end{tabular}

Table 6

Logistic regression analysis of HDP and lipid levels

\begin{tabular}{|lllll|}
\hline Factors & HDP & & & \\
\cline { 2 - 5 } & B & P & OR & $95 \%$ Cl \\
\hline TG & 0.071 & 0.849 & 1.073 & $0.519 \sim 2.220$ \\
\hline TC & -0.139 & 0.454 & 0.870 & $0.605 \sim 1.252$ \\
\hline HDL & -0.066 & 0.934 & 0.936 & $0.193 \sim 4.532$ \\
\hline LDL & -0.267 & 0.283 & 0.766 & $0.471 \sim 1.246$ \\
\hline Apo A & 0.108 & 0.805 & 1.114 & $0.472 \sim 2.632$ \\
\hline Apo B & -0.125 & 0.832 & 0.883 & $2.278 \sim 2.800$ \\
\hline AIP & 1.496 & 0.572 & 4.464 & $0.025 \sim 805.288$ \\
\hline
\end{tabular}

\section{Discussion}

Maternal lipid metabolism changes apparently throughout gestation. During the $1 \mathrm{st}$ two-thirds of gestation, there is an elevation in fat accumulation, related to hyperphagia and increased lipogenesis [10, 11]. In the last 3rd trimester of gestation, maternal fat storage decreases due to the enhanced lipolytic activity and declined lipoprotein lipase activity $[12,13]$. These alterations are reflections of maternal 
physiologic adaptation to energy demand of the fetus, as well as preparations for delivery and lactation [14]. Gestational dyslipidemia, an abnormal elevation of lipids, has been associated with adverse pregnancy complications, including GDM and HDP. Although most studies show that circulating lipid patterns are different between GDM and normal pregnancy, results have been inconsistent [15]. It has been reported that patients with GDM have increased concentrations of TG, TC and LDL-C and lower levels of HDL-C $[16,17]$. However, other studies indicate that no elevated serum TC and LDL-C levels were found in the 1st, 2nd, and 3rd trimesters, between patients with GDM and normal pregnant women [18]. Furthermore, there is no changes of TG levels in GDM group compared to non-diabetic group [19]. The $\mathrm{TG} / \mathrm{HDL}-\mathrm{C}$ ratio is higher in GDM compared with normal pregnancy $[20,21]$. In present study, we found that women with GDM had higher levels of TG, AIP and lower levels of TC and HDL-C than that of control. We further revealed that the incidence of GDM was positively correlated with levels of TG and AIP, while negative correlated with HDL-C. Thus, our data suggested abnormal maternal lipids have a role in the pathogenesis of GDM.

HDP can be classified by the terms chronic hypertension, gestational hypertension (GH), preeclampsia (PE) or chronic hypertension with superimposed PE [22]. Evidence suggests that HDP is closely related to lipid profile abnormalities, however, data still are conflicting. Contini et al reported that high concentrations of TG are a feature of PE [23]. A meta-analysis demonstrates that patients with PE have increased levels of TG and TC through pregnancy, as well as lower levels of HDL-C in the third trimester [24]. Other studies do not found any differences of neither TG nor TC levels between PE and uncomplicated pregnancies [25]. Our results illustrated that compared with the control group, the HDP group showed higher TG concentrations and AIP in the second trimester. Furthermore, AIP was correlated positively with the incidence of HDP. Collectively, these data suggested that lipids in women with HDP showed a more metabolic condition than the physiological requirement.

Although changes in lipid profile are expected, it is still difficult to determine the cut-off level for diagnosing hyperlipidemia in pregnant women. We combined the diagnostic criteria of dyslipidemia in general Chinese population and clinical data during pregnancy, pregnant women whose TC > $6.20 \mathrm{mmol} / \mathrm{L}$ and $T \mathrm{G}>2.30 \mathrm{mmol} / \mathrm{L}$ or to either of them were diagnosed with hyperlipidemia. Expect for pregnancy complications [26], maternal dyslipidemia has been proved to be closely linked with adverse pregnancy outcomes including cesarean delivery and postpartum hemorrhage [27]. Consistent with previous researches, we reported that women with hyperlipidemia compared with these in normal lipid group had a higher rate of GDM and cesarean section. In addition, the mean of postpartum hemorrhage both in the GDM and HDP groups was greater than that in the control group. As for the conditions of newborn, evidence shows that dyslipidemia results in an adverse intrauterine environment and can induce excessive fetal growth. Recently, some maternal lipid parameters have been served as independent predictors of fetal overgrowth, especially in women complicated by $\operatorname{GDM}[28,29]$. The risk of macrosomia was positively related to TG levels, while negatively related to HDL-C levels in non-diabetic pregnancies [9]. However other studies failed to find any association [30, 31]. In the present study, we found that the incidence of macrosomia was significantly increased in the hyperlipidemia group. Meanwhile, the average body length and weigh of newborn in the GDM and HDP groups were 
significantly lower than those of the control group. Recognizing lipid abnormalities may allow for appropriate risk-reducing interventions of pregnancy complications as well as clinical outcome of mother and fetus.

The associations of increased levels of lipids during gestation with the risk of GDM and HDP have not been clarified $[8,18,32,33]$. We then to explore whether dyslipidemia in the 2 nd trimester has potential clinical utility for identifying women at risk for developing GDM and HDP. Logistic regression analysis showed that AIP was a risk factor of GDM, while TC was a protective factor. Among the Chinese population, Jin et al report that lower levels of HDL-C have a significant association with increased risk of GDM [26]. Our results illustrated that the incidence of GDM was negatively correlated with levels of TC and HDL-C. TC reflects the content of total cholesterol including HDL-C and LDL-C. Taken together, we considered that the proper increase of HDL-C so-called "good" cholesterol, had a protective effect for those with GDM.

The present study was limited by its retrospective design and some possible confounders such as weight and lipid profiles at all gestational stages could not be accounted. A prospective observational design is needed to overcome the limitation of lacking other parameters and corroborate our findings.

\section{Conclusion}

In conclusion, this study adds to the current knowledge of associations between maternal lipid concentrations during pregnancy and outcomes for the mother and newborn. More research is warranted to establish the standard of hyperlipidemia diagnosis during pregnancy according to local maternal characteristics. As for pregnant woman with abnormal blood lipids, close monitoring and diet management should be carried out to prevent pregnancy complications and improve clinical outcomes as much as possible.

\section{Abbreviations}

Apo A1/B

Apolipoprotein A1/B

$\mathrm{GH}$

Gestational hypertension

PE

Preeclampsia

AIP

Atherogenic index of plasma

BMI

Body mass index

GDM

Gestational diabetes mellitus 
HDP

Hypertensive disorders of pregnancy

HDL-C

High-density lipoprotein-cholesterol

LDL-C

Low-density lipoprotein-cholesterol

TC

Total cholesterol

TG

Triglycerides

\section{Declarations}

Ethics approval and consent to participate

Since this was a retrospective study, based only on anonymous data analysis, informed consent was not required (BMC Pregnancy Childbirth. 2020 Apr;20:246). The Medical Ethics Committee of Nanjing Drum Tower Hospital ruled that no formal ethics approval was required in this particular case.

Consent for publication

Not applicable.

Availability of data and materials

The datasets used and analyzed during the current study are available from the corresponding author on reasonable request.

Competing interests

The authors declare that they have no competing interests.

Funding

This work was supported by the Key Project supported by Medical Science and Technology Development

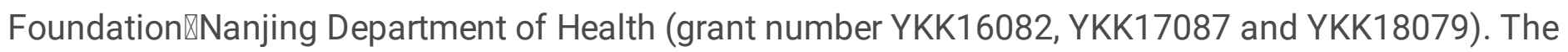
funds mainly been used for the study analysis, interpretation of data and writing the manuscript. The authors declare that there are no conflicts of interest.

Authors' contributions

YL participated in data acquisition, statistical analysis, literature search and drafted the manuscript. WQC and XQW contributed to the acquisition of the data. FJJ and XTC contributed to the analysis and interpretation of the data. All authors read and approved the final manuscript. 
Acknowledgements

Not applicable

\section{References}

1. Ryckman KK, Spracklen CN, Smith CJ, Robinson JG, Saftlas AF. Maternal lipid levels during pregnancy and gestational diabetes: a systematic review and meta-analysis. BJOG.2015;122:643 51.

2. WHO Recommendations for Prevention. and Treatment of Pre-Eclampsia and Eclampsia. Geneva, World Health Organization, 2011.

3. Gaillard R, Durmus B, Hofman A, Mackenbach JP, Steegers EA, Jaddoe VW. Risk factors and outcomes of maternal obesity and excessive weight gain during pregnancy. Obesity (Silver Spring). 2013;21:1046-55.

4. Guariguata L, Whiting DR, Hambleton I, Beagley J, Linnenkamp U, Shaw JE. Global estimates of diabetes prevalence for 2013 and projections for 2035. Diabetes Res Clin Pract. 2014;103:137-49.

5. Ray JG, Diamond P, Singh G, Bell CM. Brief overview of maternal triglycerides as a risk factor for preeclampsia. BJOG.2006;113:379 - 86.

6. Ryckman KK, Spracklen CN, Smith CJ, Robinson JG, Saftlas AF. Maternal lipid levels during pregnancy and gestational diabetes: a systematic review and meta-analysis. BJOG.2015;122:643 51.

7. Emet T, Ustuner I, Guven SG, Balik G, Ural UM, Tekin YB, Senturk S, Sahin FK, Avsar AF. Plasma lipids and lipoproteins during pregnancy and related pregnancy outcomes. Arch Gynecol Obstet. 2013;288:49-55.

8. Shen H, Liu X, Chen Y, He B, Cheng W. Associations of lipid levels during gestation with hypertensive disorders of pregnancy and gestational diabetes mellitus: a prospective longitudinal cohort study. BMJ Open. 2016;6:e13509.

9. Wang X, Guan Q, Zhao J, Yang F, Yuan Z, Yin Y, Fang R, Liu L, Zuo C, Gao L. Association of maternal serum lipids at late gestation with the risk of neonatal macrosomia in women without diabetes mellitus. Lipids Health Dis. 2018;17:78.

10. Murphy SP, Abrams BF. Changes in energy intakes during pregnancy and lactation in a national sample of US women. Am J Public Health. 1993;83:1161-3.

11. Mankuta D, Elami-Suzin M, Elhayani A, Vinker S. Lipid profile in consecutive pregnancies. Lipids Health Dis. 2010;9:58.

12. Herrera E, Lasuncion MA, Gomez-Coronado D, Aranda P, Lopez-Luna P, Maier I. Role of lipoprotein lipase activity on lipoprotein metabolism and the fate of circulating triglycerides in pregnancy. Am $\mathrm{J}$ Obstet Gynecol. 1988;158:1575-83. 
13. Meyer BJ, Stewart FM, Brown EA, Cooney J, Nilsson S, Olivecrona G, Ramsay JE, Griffin BA, Caslake MJ, Freeman DJ. Maternal obesity is associated with the formation of small dense LDL and hypoadiponectinemia in the third trimester. J Clin Endocrinol Metab. 2013;98:643-52.

14. Di Cianni G, Miccoli R, Volpe L, Lencioni C, Ghio A, Giovannitti MG, Cuccuru I, Pellegrini G, Chatzianagnostou K, Boldrini A, Del PS. Maternal triglyceride levels and newborn weight in pregnant women with normal glucose tolerance. Diabet Med. 2005;22:21-5.

15. Herrera E, Ortega-Senovilla H. Disturbances in lipid metabolism in diabetic pregnancy - Are these the cause of the problem? Best Pract Res Clin Endocrinol Metab. 2010;24:515-25.

16. Savvidou M, Nelson SM, Makgoba M, Messow CM, Sattar N, Nicolaides K. First-trimester prediction of gestational diabetes mellitus: examining the potential of combining maternal characteristics and laboratory measures. Diabetes. 2010;59:3017-22.

17. Li G, Kong L, Zhang L, Fan L, Su Y, Rose JC, Zhang W. Early Pregnancy Maternal Lipid Profiles and the Risk of Gestational Diabetes Mellitus Stratified for Body Mass Index. Reprod Sci. 2015;22:712-7.

18. Wang J, Li Z, Lin L. Maternal lipid profiles in women with and without gestational diabetes mellitus. Med (Baltim). 2019;98:e15320.

19. Montelongo A, Lasuncion MA, Pallardo LF, Herrera E. Longitudinal study of plasma lipoproteins and hormones during pregnancy in normal and diabetic women. Diabetes. 1992;41:1651-9.

20. Gasevic D, Frohlich J, Mancini GB, Lear SA. The association between triglyceride to high-densitylipoprotein cholesterol ratio and insulin resistance in a multiethnic primary prevention cohort. Metabolism. 2012;61:583-9.

21. Khosrowbeygi A, Shiamizadeh N, Taghizadeh N. Maternal circulating levels of some metabolic syndrome biomarkers in gestational diabetes mellitus. Endocrine. 2016;51:245-55.

22. Tranquilli AL, Dekker G, Magee L, Roberts J, Sibai BM, Steyn W, Zeeman GG, Brown MA. The classification, diagnosis and management of the hypertensive disorders of pregnancy: A revised statement from the ISSHP. Pregnancy Hypertens. 2014;4:97-104.

23. Contini C, Winkler BS, Maass N, Alkatout I, Winkler K, Pecks U. Concomitant intrauterine growth restriction alters the lipoprotein profile in preeclampsia. Pregnancy Hypertens. 2019;15:154-60.

24. Spracklen CN, Smith CJ, Saftlas AF, Robinson JG, Ryckman KK. Maternal hyperlipidemia and the risk of preeclampsia: a meta-analysis. Am J Epidemiol. 2014;180:346-58.

25. Hentschke MR, Poli-de-Figueiredo CE, Da CB, Kurlak LO, Williams PJ, Mistry HD. Is the atherosclerotic phenotype of preeclamptic placentas due to altered lipoprotein concentrations and placental lipoprotein receptors? Role of a small-for-gestational-age phenotype. J Lipid Res. 2013;54:2658-64.

26. Jin WY, Lin SL, Hou RL, Chen XY, Han T, Jin Y, Tang L, Zhu ZW, Zhao ZY. Associations between maternal lipid profile and pregnancy complications and perinatal outcomes: a population-based study from China. BMC Pregnancy Childbirth. 2016;16:60.

27. Mohsenzadeh-Ledari F, Taghizadeh Z, Motaghi Z, Keramat A, Moosazadeh M, Najafi A. Appropriate Interventions for Pregnant Women with Indicators of Metabolic Syndrome on Pregnancy Outcomes: A Systematic Review. Int J Prev Med. 2019;10:2. 
28. Schaefer-Graf UM, Graf K, Kulbacka I, Kjos SL, Dudenhausen J, Vetter K, Herrera E. Maternal lipids as strong determinants of fetal environment and growth in pregnancies with gestational diabetes mellitus. Diabetes Care. 2008;31:1858-63.

29. Son GH, Kwon JY, Kim YH, Park YW. Maternal serum triglycerides as predictive factors for large-forgestational age newborns in women with gestational diabetes mellitus. Acta Obstet Gynecol Scand. 2010;89:700-4.

30. Retnakaran R, Ye C, Hanley AJ, Connelly PW, Sermer M, Zinman B, Hamilton JK: Effect of maternal weight, adipokines, glucose intolerance and lipids on infant birth weight among women without gestational diabetes mellitus. CMAJ.2012;184:1353-60.

31. Parlakgumus HA, Aytac PC, Kalayci H, Tarim E. First trimester maternal lipid levels and serum markers of small- and large-for-gestational age infants. J Matern Fetal Neonatal Med. 2014;27:4851.

32. Enquobahrie DA, Williams MA, Butler CL, Frederick IO, Miller RS, Luthy DA. Maternal plasma lipid concentrations in early pregnancy and risk of preeclampsia. Am J Hypertens. 2004;17:574-81.

33. Lima VJ, Andrade CR, Ruschi GE, Sass N. Serum lipid levels in pregnancies complicated by preeclampsia. Sao Paulo MedJ. 2011;129:73-6. 